\title{
The problematic of language in Assia Dejebar's Strasbourg Nights
}

Dilber ZEYTINKAYA *

* Marmara Üniversitesi

E-mail: dilber.zeytinkaya@marmara.edu.tr

Copyright (C) 2015 Dilber ZEYTINKAYA. This is an open access article distributed under the Eurasian Academy of Sciences License, which permits unrestricted use, distribution, and reproduction in any medium, provided the original work is properly cited.

\begin{abstract}
Assia Djebar's 'Strasbourg Nights' formed on the problem of language in her work on this study, the search for identity, the preferred language of love, focuses on the theme of silence caused by the language. The aim of this study is to stimulate thinking on the question of language processing. Djebar who was freed through the life of the French language has a great importance. Handle the language issue is in the works she wrote. Her work go from Strasbourg between languages and cultures, languages, religions, races, cultures are subject to different pairs from each other. Literary criticism with an analytical method to perform and you will find answers to the following questions: Couples communicate in which language? Is it possible to love your enemies, even if they speak the other language? Does culture affect the culture or language affects the language study? Couples want to impersonate another person? Which is trying to find a common point of different nationalities and couples living in nomad is seeking peace through language. Examined study, languages other than one language, one nation from other nations, has set up a bridge in the transition from one culture to another culture.
\end{abstract}

Keywords: Djebar, Strasbourg Nights, language, identity, multiculturalism

\section{La question de la langue chez Les Nuits de Strasbourg d'Assia Djebar}

\section{ÖZET}

Assia Djebar'ın 'Strasbourg Geceleri' adlı eserindeki dil sorunsalı üzerine şekillenen bu çalışmada, kimlik arayışı, aşkta tercih edilen dil, dilin neden olduğu sessizlik temalarının üzerinde durulmaktadır. Çalışmanın amacı, dil sorunsalı üzerinde düşünmeye teşvik etmektir. Fransızca aracılığıyla özgürlüğüne kavuşan Djebar'ın yaşamında dil, büyük bir öneme sahiptir. $\mathrm{Bu}$ nedenle yazmış olduğu eserlerde dil sorununu ayrıntısıyla işlemektedir. Diller ve kültürler arasında gidip gelen Strasbourg Geceleri adlı eseri, dilleri, dinleri, 1rkları, kültürleri birbirinden farklı çiftleri konu etmektedir. Analitik yöntemle edebiyat eleştirisi gerçekleştirecek ve şu sorulara yanıt aranacaktır : Çiftler hangi dilde iletişim kurmaktadır? Düşman dahi olsa ötekinin dilinde aşk mümkün müdür ? Hangi dili konuşacaklarını belirleyen çiftler midir yoksa dil mi çiftleri yönlendirmektedir ? Kültürü etkileyen dil midir yoksa kültür mü dili etkilemektedir ? Çiftler kendileri gibi mi kalmak isterler yoksa başka kişiliklere mi bürünmek isterler ? Farklı milletlere mensup ve göçebe yaşamlarında ortak bir nokta bulmaya çalışan çiftler, dil aracılığıyla barış arayışındadır. İncelenen eser, bir dilden diğer dile, bir milletten diğer millete, bir kültürden diğer kültüre geçişlerde köprü kurmaktadır.

Anahtar Kelimeler: Djebar, Strasbourg Geceleri, dil, kimlik, çokkültürlülük 


\section{Introduction}

Ce travail est centré sur l'œuvre d'Assia Djebar qui s'intitule Les Nuits de Strasbourg. On va l'analyser en abordant le thème "langue" car la problématique du langage occupe une place centrale. A travers la langue, on va se pencher sur la problématique des nationalités, de l'amour, du silence, et du rêve. Il est possible de préciser que le livre Les Nuits de Strasbourg est à la fois sur le nomadisme et sur l'exode. Avant de passer à l'analyse, on doit connaitre Djebar. Il faut retourner au passé afin de mieux comprendre son œuvre, son histoire et son écriture.

Assia Djebar est née le 4 août 1936, dans la petite ville de Cherchell sur le bord de la mer en Algérie. Ses parents l'ont nommée Fatime-Zohra Imalayen. Comme jeune fille, elle est allée à l'école primaire où son père s'enseignait le français. Après, elle a commencé à l'école privée avec l'internat près de la capitale d'Algérie pour le lycée. En 1955, Djebar était un des premières femmes qui étaient acceptés à l'Ecole Normale Supérieure à Paris. Son père l'a encouragé de poursuivre ses études et d'aller à Paris. Pendant son séjour en France, elle s'est engagée au mouvement de libération algérien et aux grèves des étudiants. La libération était prés de son cœur pour plus de raisons qu'un, depuis que son frère était tenu dans une prison française pendant cette longue lutte. ${ }^{1}$

On peut énumérer ses œuvres et leurs traductions en turc: La Soif, (1957; Susuzluk); Les impatients, (1958; Sabırsızlar); Les Enfants du Nouveau Monde, (1962; Yeni Dünyanın Çocukları); Les Alouettes naïves, (1967; Saf Tarlakuşları); Poème pour une algérie heureuse, (1969; Mutlu Bir Cezayir İçin Şiirler); Rouge l'aube (1969; Kızıl Şafak); L'Amour, la fantasia, (1985; Aşk ve Fantazya); Ombre sultane 1987; Loin de Médine, 1991; Vaste est la prison, 1995; Le blanc de l'Algérie, 1996.; Oran, langue morte, 1997; Les Nuits de Strasbourg, 1997; Femmes d'Alger dans leur appartement, 2002; La femme sans sépulture, 2002; La disparition de la langue française, 2003; "Nulle part dans la maison de mon père", 2008.

Afin de mieux analyser l'œuvre Les Nuits de Strasbourg, il faut savoir les raisons que Djebar choisit le lieu en tant que Strasbourg. Tout d'abord, c'est un lieu occidental, ensuite c'est un lieu expressif pour écrire ses œuvres. On ne le considère qu'un simple lieu, Strasbourg est aussi l'un des personnages principaux. Strasbourg est une ville frontière et déchirée par la guerre entre la France et l'Allemagne. C'est une ville des passages et de confluences. C'est l'endroit idéal pour la rencontre des différentes cultures. La ville est placée au centre de

\footnotetext{
${ }^{1}$ http://sites.duke.edu/globalfrance/assia-djebar/
} 
l'Europe, c'est la raison qu'on le considère comme un carrefour important. C'est pour cette raison que c'est une ville où se croisent plusieurs langues et cultures.

Il est utile de préciser que le langage est l'un des thèmes fondamentaux qui joue un rôle essentiel dans cet œuvre. Pour pouvoir analyser l'œuvre sous l'aspect de la question du langage, il nous faut d'abord éclairer la notion du langage chez Djebar. La langue est indispensable pour elle et occupe une place centrale dans sa vie. C'est grâce à la langue française qu'elle ne sera pas enfermée comme beaucoup d'autres filles algériennes et elle gagnera sa liberté.

La langue est la problématique majeure comme on avait déjà indiqué. L'Alsace et L'Algérie sont des deux territoires où l'on parle plusieurs langues en ce qui concerne l'Alsace : le français, l'allemand, l'alsacien; et en ce qui concerne L'Algérie : l'arabe, le berbère, le français. Suite à la guerre franco-allemande, il est en question de plusieurs langues. Ces langues vous orientent. On y observe la guerre des langues. C'est pour cette raison qu'on essaie de donner les exemples qui prouvent que c'est bel et bien la guerre des langues. Puisque c'est un livre entre les langues et les cultures, l'œuvre met en avant l'importance de la langue.

La langue est aussi liée à l'amour. Il existe les couples qui se composent d'origines, de cultures et de religions différentes ainsi que Thelja et François ; Eve et Hans ; Irma et Karl ; Jacqueline et Ali. Thelja est algérienne mais François est français. Eve est juive mais Hans est allemand. Irma est juive mais Karl est alsacien. Jacqueline est alsacienne mais Ali est algérien. Après avoir passé neuf nuits avec François, Thelja, le personnage principal, disparaît subitement pour réapparaître une nuit, six mois plus tard à Strasbourg. Est-ce la disparition ou bien la renaissance ? En quelle langue communiquent les couples ? Est-ce que l'on peut aimer dans la langue de l'autre même s'il est un ennemi ? On doit signaler que cet essai va répondre à ces questions et aborde la question du langage chez Les Nuits de Strasbourg.

A la lumière de tout ce qu'on vient d'exprimer jusqu'ici, il me semble susceptible d'orienter la problématique du langage. Dans ce travail, on va aborder la méthode analytique et critique littéraire. L'objectif est de faire penser les lecteurs à propos de la problématique.

\section{La question de la langue chez Eve et Hans}

Le prénom « Hans » nous montre que l'homme mentionné est vient d'une nationalité différent par rapport à la femme. On n'entend pas son prénom jusqu'au milieu de l'œuvre. Eve a peur de dire tout haut son prénom, elle ne peut pas crier à cor et à cri son amour à cause de l'impossibilité. On y rencontre la trace de l'impossibilité de l'amour, car il s'agit de la guerre imperceptible entre Eve et Hans. Elle n'arrive même pas à avouer en elle-même. La guerre entre la France et L'Allemagne se voit comme un obstacle même un piège pour leur relation 
amoureuse. L'amour entre une française et un allemand se voit aussi dangereux qu'elle se combat et se démène pour s'en sortir. C'est comme la recherche d'un remède : leur amour peut adoucir les traumatismes que la guerre cause :

«Elle a prononcé ce prénom “Hans”, je vous ai aussitôt interpellé en moi : Eve qui croyait avoir franchi l'impossible, l'interdit fiché en elle depuis l'enfance, Eve ma plus proche, nomme pourtant son amour I... Or moi (je vous parle, et je vous le dirai sans doute ce soir), je ne peux dire tout haut, ni même en moi, votre nom... Pourquoi ? Si longtemps après la guerre - je précise "la guerre chez moi entre les vôtres et les miens". (p.78)

Eve a une idée de ne jamais partir en Allemagne même pas pour un jour mais elle est tombée amoureuse d'un homme allemand. On y observe la question du langage qui cause un conflit intérieur. Elle refuse d'aller en Allemagne parce que c'est un endroit où les juifs sont morts. La langue allemande représente la langue-conflit :

"- “Jamais, jamais, moi née d'un père juif andalou et de mère juive berbère, jamais je ne mettrai les pieds en Allemagne. Même pas pour un jour ! Me préserver !...”»(p.68)

"Voici qu'à la suite d'un “coup de foudre”, je me retrouve au cour même de “ma” zone interdite, pour ainsi dire en terrain ennemi... Alors, j'ai tourné, tourné, je n'ai plus su où j'en étais !... J'ai répété : “pas l'Allemagne !’’... » (p.68)

La nationalité est une notion qui peut engendre quelques problèmes entre les couples. L'amour est considéré comme un obstacle devant l'homme qui est allemand. Il vaut mieux de franchir les obstacles pour construire la véritable relation et fournir l'échange jusqu'à ce qu'on a véritable communication :

«- Pourquoi mon “dernier amour” ? continuais-tu, inscrivant les questions à ma place.

-Le dernier, parce qu'il s'agit d'un homme allemand !» (p.67)

Eve ne préfère pas, elle refuse de parler l'allemand même si elle a appris au lycée. C'est peutêtre qu'elle défend la notion de ne pas parler la langue de l'ennemi parce qu'on le voit comme un ennemi. Son choix nous montre qu'elle ne voudrait pas trahir son identité, son peuple et sa langue. Son refus est respecté par Hans. Par contre, il apprend l'arabe marocain qui est la langue maternelle d'Eve : 
« Je trouverai du travail dans cette ville. Il faut qu'il apprenne un peu le français... Moi, je ne lui parle pas dans «sa» langue. (Tu le sais, toi, que j’ai appris au lycée l'allemand. Par défi.) Mais je ne parlerai pas avec lui cette langue. » (p.69)

Hans s'efforce d'apprendre la langue de l'autre, c'est-à-dire, la langue d'Eve. Elle ressent à la fois l'enfer et le paradis. C'est en même temps pour la mémoire et pour la volupté.

«-Il s'est mis à étudier le français avec méthode; il dit qu'il apprendra ensuite le dialecte... Moi, je me donne. J'ai tout joué d'un coup. Je suis en enfer et en paradis («en enfer» pour la mémoire, «en paradis» pour la volupté)» (p.70)

Leur relation est restreinte. Ils communiquent en utilisant quelques peu mots en français et en anglais. Ils n'arrivent pas à rassembler et unir au même point. Il vaut mieux qu'ils trouvent un point d'équilibre :

«Quand nous n'avions pas tellement de mots à échanger : toi, dix mots français, et moi, deux ou trois fois plus... en anglais. Tu sifflais alors ; moi, souvent, je te suivais en fredonnant. Ainsi, tu fais appel à la complicité du début, de l'année dernière. » (p.93)

Eve le voit comme un étranger absolu parce qu'il est d'une autre nationalité et il parle une autre langue que celle d'Eve. Elle se contente de l'appeler « étranger» au lieu de l'appeler par son prénom qui rappelle la guerre et qui provoque le traumatisme. Elle se contente de l'appeler « étranger » :

" "L'étranger absolu, songea-t-elle, demain, ce sera Hans. Lui, mon amour allemand que ne pourra s'empêcher de me reprocher mon cousin, à leur déjeuner du samedi !” »(p.112)

On y voit qu'Hans s'efforce d'apprendre la langue arabe. Il connait déjà quelques mots mais il voudrait enrichir son lexique. D'ailleurs il prononce les mots français pour apprendre l'arabe. Il est possible de préciser qu'il a envie d'apprendre les langues différentes :

«-Tu attends la leçon ? demande Hans, en prononçant lentement ses mots français.

Ils commencent, comme souvent les samedis, leur dialogue le plus souvent mimé.

Keib / articule-t-elle, et, d'emblée, elle rit...

Hans connaît le mot kelb ; la conversation débute par le plus facile...:

- $\quad$ El oueld! el bent ! 
- $\quad$ Trop simple ! proteste Hans amusé. Moi, je connais tout ça,., le fils, la fille, le père, la mère... Dis-moi une phrase entière ! » (p.139)

Hans ne peut pas utiliser le français comme il faut, il est certain qu'il est de niveau débutant car il ne peut pas conjuguer les verbes, il les utilise à l'infinitif. Donc, son savoir par rapport à la conjugaison n'est pas suffisant. Ses phrases ne concernent pas les articles ; on y observe encore une fois le manque de savoir par rapport à la langue française :

«Ensuite, dans son français coupé menu, haché, sans article le plus souvent et avec le verbe souvent à l'infinitif, dans son français niveau début d'école primaire, Touma, la dame de soixante ans, éprouve le besoin de raconter. Assez vite, Hans entre dans le rythme entrecoupé, suit l'écoulement et la fièvre des souffrances passées. » (p.140)

Eve parle avec Mina dans sa langue pour qu'elle ne ressente pas le vide. Le vide en Mina est essaie de substituer par Eve. Alors qu'Eve agit comme la mère de Mina afin qu'elle subisse le moindre dégât. Par la voie de langue, on essaie de garder la communication et de réduire les souffrances :

«Comme toi, s’attendrit Touma. La mère de Mina, marocaine : Ourdia. Mina parle marocain. Moi, avec elle, je parle comme elle. Comme sa mère... (Elle s'absente, semble souffrir, sa voix recherche soudain.) Sa mère, partie avec un Français. Ali m’a donné Mina. Mina, ma ..... » (p.141)

Hans préfère parler l'arabe quand il est nerveux, quand il voudrait qu'Eve lui comprenne. On y voit que la langue est utilisée pour fortifier leur liaison. Il sert à fumer le calumet de la paix afin que les couples se réconcilient :

"Hans voudrait s'exclamer : “Ça suffit ! Arrête !” Il trouve soudain les mots arabes yakfi, yakfi, Lalla /"; il les lui dirait d'une voix grave, d'une voix désespérée. » (p.144) 
Mina n'a pas envie de parler français même si elle comprend la langue. Bien qu'elle comprenne tout, elle répond dans l'arabe. Elle préfère utiliser la langue alsacienne. Bien que Hans refuse de parler dans la langue d'Eve, il conversait en arabe marocain avec Mina :

«Etrange également, et Hans ne sait pas que je le sais, un autre samedi où, en arrivant, je les trouvais tous deux, devine en quelle langue ils conversaient, sans se douter que je m'étais arrêtée derrière la porte entrouverte : en arabe marocain, mais oui, Thelja ! Mina ne parle pas, ou ne veut pas parler français, même à l'école, me dit-on. Elle comprend tout : elle répond dans l'arabe de sa mère ; quelquefois, dans la cour en bas ou devant les escaliers, je l'ai surprise aussi en train de bavarder avec les enfants des voisins : mais en alsacien !.., Donc, ce samedi-là, j'entends Hans répéter après la fillette - celle-ci devenant son professeur quand il vient pour le weekend - des listes de mots... coüncidence, dans la langue de mon premier mari, de ma fillette restée là-bas ! »(p.146-147)

Hans déclare qu'il fait un effort pour apprendre la langue française même apprenant son dialecte. Hans pratique aussi la langue arabe. Par contre, la raison qu'il préfère parler la langue arabe, c'est en raison d'un amour secret. On y voit que la langue est liée à l'amour :

«- Je vais faire des progrès en français, je promets ! (Et sur ce point, depuis ce moment-là, il a fait mieux que des progrès !) Mais il avait ajouté : je vais même me mettre au dialecte !...

J'entends encore la vivacité de son exclamation. Moi, naturellement, j'avais cru qu'il pratiquerait ensuite l'alsacien, ce qui, pour lui, allemand, serait plus facile !... Mais non, tu le vois, Thelja, et j'en suis encore toute troublée, c'était de l'arabe dont il parlait.,. Pourquoi ? Je ne sais encore... » (p.147)

Après la dispute, Hans parle allemand pour se rapprocher d'Eve parce que les mots dits dans la langue maternelle sont plus efficients : 
«- Chut !... recommande-t-il. Ne parle plus. L'oubli vient vite, et il ajoute, dans un murmure, trois mots ou quatre d'un vers allemand. » (p.165)

La question de la religion cause un grand problème entre Eve et Hans. Le chrétien se moque d'une juive. Il se moque d'elle. A part la problématique de la langue, la religion se voit comme un obstacle entre les deux. Au moment de la dispute, Hans préfère l'anglais car c'est une langue neutre. En utilisant le mot «my love» il passe par un terrain neutre, un terrain d'espoir affirmant qu'il n'est pas le Christ. Pour calmer la situation, Hans utilise l'anglais parce que cette langue neutre ne concerne pas de souffrances et de douleurs :

«... si c'est un garçon, je le fais circoncire !...

D’ailleurs, lui, l'homme allemand, il a dit : “en bonne mère juive”. Il a osé le dire.

Il a osé ? » (pp.160-161)

«- Tu veux frapper sur l'autre joue, maintenant ?... je ne suis pas le Christ, "my love"!

Il a terminé en anglais, au moins un terrain neutre, un minuscule espace, un tout petit terre-plein d'espoir, "my love”, deux mots passe-partout, voletant d'une autre rive...

Elle a perçu l'ironie : "je ne suis pas le Christ !"... Elle a compris : “frappe encore, frappe donc ! My love, my sweet love!...” «(p.163)

Le serment mentionné est la trace de Français écrit. Par le biais de serment, Eve et Hans s'unissent dans la fraternité. Serments de Strasbourg est le serment entre Louis le Germanique et Charles le Chauve contre leurs frères Lothaire, par lequel les deux armées expriment non seulement leurs volontés de faire la paix mais aussi leur acceptation pacifique de la langue et de la culture de l'autre. Dans Les nuits de Strasbourg, le serment est lié à la langue :

«Il s'agit bien d'un serment !

-D’un serment? interrogea à nouveau Hans - il hésita une seconde sur le sens exact du mot français.

Alors Eve, le visage rougi d'animation, devint intarissable : 
Le serment de Strasbourg, tu sais ce que c'est?... Non, tu ne vois pas, ou tu as oublié... C’est votre histoire pourtant : celle de cette ville, la tienne aussi puisque tu es allemand, celle des Français ; moi, en tout cas, il y a des siècles de cela, je n'étais pas française, ni allemande, ni même de ce nord de l'Europe, mes ancêtres, en Afrique du Nord, parlaient berbère, pratiquaient le judaïsme depuis très longtemps,... » (p.234)

Eve dit qu'il s'agit d'un "acte politique", le serment appartient à la fois à l'histoire des français et des allemands. C'est un serment qui unit les parties opposés. C'est l'exemple d'un échange linguistique. Pour leur relation sensuelle, c'est plutôt un serment d'amour :

« L'important... l'important aujourd'hui, l'important pour nous... C'est que, voistu, leur serment d'alliance (et pour nous, c'est un serment d'amour plutôt), l'important pour moi aujourd'hui est que Louis, l'aîné, va prononcer le serment en français, ou plus exactement en roman, dans la langue du frère, et que Charles le Français va l'épeler, lui, dans la langue tudesque, la langue de l'autre... Et voistu, les deux armées font pareil, chacune va reprendre le serment dans la langue de l'autre armée... de l'autre chef... C'est un acte politique, c'est surtout un échange linguistique!»(p.236)

Hans et François se mettent à parler l'allemand entre eux. Bien que François soit français, il accepte de parler la langue de l'autre. L'autre langue est considérée comme une langue de l'ennemi. La langue est perçue comme un obstacle, il faut franchir les pièges, il faut les écarter afin qu'on survive :

«Hans le rejoignit. Les deux hommes se mirent à dialoguer un peu bas, en allemand. » (p.177)

Eve comprend qu'elle doit parler l'allemand avec Hans. C'est pour cette raison qu'elle cesse de s'obstiner et finit par parler la langue de l'autre:

«- Je suis prête, ô Hans, prête aujourd'hui à te parler enfin dans ta langue... » (p.236). 
Eve avait promis de ne pas parler l'allemand, même de ne pas rentrer en Allemagne. Pourtant, ils font un serment en ce qui concerne la langue. Eve jure en langue germanique et cherche un compromis pour vivre son histoire avec Hans :

«Eve donc, la voix un peu étouffée, commença en langue germanique le même serment ; sa voix peu à peu s'éclaircit, elle ne déforma rien des consonances, ni du rythme de la langue de Hans qui l'écoutait, ému » (p.237)

Pour fournir l'information, il faut clarifier la communication. Afin que les gens s'entendent, il faut la traduction des mots inconnus. La langue est considérée comme un médiateur.

"L'un des chauffeurs de taxi accepte en lui disant : "el moût ?" il a un geste désinvolte du bras et ajoute : "el Koul en moût!” Son prix est raisonnable ;...

- $\quad$ Rien de plus simple : j'ai trouvé un taxi d'une compagnie de là-bas. On appelle ce taxi "el koul en moût" !

Il finit par lui traduire les mots arabes :

- L L L L chauffeur de taxi vous a dit, mademoiselle : "si nous devons mourir, nous mourrons tous!” "

(p.253)

Les noms désignent quelques indices par rapport aux nationalités des gens. Malgré cela, on ne peut pas être certain si c'est un français ou bien allemand. Il faut retourner en arrière et comprendre que les gens subissent quelles circonstances afin de parler même une seule langue :

«Quelques années après mon arrivée aux Etats-Unis, où, sur une remarque pincée de mon directeur de thèse, quand par hasard je dus dire que, malgré mon nom français, j'étais fille de juifs persécutés en Alsace, ce professeur américain me dit sèchement :

- Quand donc apprendrez-vous l'hébreu ? Quand donc irez-vous en Israël ?

Je n'y avais jamais pensé : mes parents, parlant allemand, qui devaient s'aimer dans cette langue, cela tombait bien, j'avais un faible pour la littérature allemande contemporaine !... Les deux années qui suivirent, j’appris l'hébreu. Puis l'été, j'allais en Israël, en vacances ! » (p.302) 
On saisit qu'il s'agit de la guerre des trois langues : le français, l'allemand et l'alsacien. Les langues dominent sur les individus. On a l'occasion de changer d'une langue à l'autre : «- "La mère amère” soliloque Irma, allant et venant d'une fenêtre à l'autre. Que demandais-je à l'inconnue, la renégate : simplement qu'elle dise tout haut mon prénom et mon nom - ou simplement, mon prénom : en français, en allemand ou en alsacien!" (p.304)

En ce qui concerne la douleur, on préfère parler la langue de l'enfance. Le fils de Touma est devenu un assassin :

«Touma ne pleurait pas. Elle m'a parlé en arabe, puis en chaoui - bien que j'aie tendance, ces dernières années, à oublier le berbère chaoui ! Elle m'a fixée et a dit, d'une voix rauque » (p.335)

En raison du colonialisme, on observe que les langues utilisées sont différentes. Elle commence à parler par la langue arabe et continue à parler la langue française avec des accents germanique et ajoutant quelques mots en alsacien :

«Elle s'est tournée vers moi, et je ne sais qui lui a dit, mais d'emblée elle m'a parlé en arabe algérien :..............

Ali, commença-t-elle, et elle parla cette fois en français, avec de temps à autre des mots étranges. Je compris, à cause de leurs consonances germaniques, qu'elle émaillait chacune de ses phrases d'un mot en alsacien.» (p.336)

Le mot alsacien weiseïkend, est utilisé pour « orphelin ». Elle utilise ce mot pour annoncer la mort de leur père. On y voit que le choix du mot différencie suivant le contexte historique:

" J'ai crié une seconde fois, et toujours sans pleurer : "orphelins !”... Je l'ai dit en alsacien ce dernier mot, à cause du médecin qui venait de sortir et qui, en dégringolant l'escalier, m'avait de la même façon apostrophée :

- Vous voici tous les deux orphelins!

Ce mot alsacien weiseïkend, je l'avais redit, pour moi toute seule.

Moi, debout, je dévidai, comme pour me venger : "weiseïkend, weiseïkend", et la pensée de mon frère ne me quittait pas.... Nous voici tous deux orphelins ! » (p.339) 
Quand on nous parle d'une langue, on vous répond de la même langue. C'est pour transmettre le bon message et recevoir le même message afin d'empêcher les malentendus :

«- Ali, soupira-t-elle, je sais que c'est à partir de cet instant qu'il m'en a voulu! (Elle s'était remise, je ne sais pourquoi, à l'arabe, parce que je l'avais approchée sans doute.) Je lui ai répondu dans la même langue : » (p.340)

Bien qu'on apprenne une langue, on ne pourrait pas toujours parler cette langue. Quelle que soit la nationalité des individus, on pourrait toujours apprendre une nouvelle langue. Par contre, apprendre ne signifie pas tout le temps qu'on parle couramment :

«Elle disait qu'enfant, alors qu'elle apprenait l'allemand au collège, elle n'avait jamais pu parler allemand avec son père... Il parlait alsacien avec sa femme, et très mal français... » (p.365)

\section{La question de la langue chez Irma et Karl}

Les langues sont considérées comme des symboles d'identité et comme marqueur d'identité.

C'est pour cette raison que la langue entretient des rapports avec la culture et l'identité :

«Il ne parlait pas français, mais anglais. Karl me traduisait. Moi, je croyais ce pays francophone. Mais ce garçon semblait n'avoir guère plus que vingt-cinq ans; c'est la génération des quarante ans, ou cinquante, qui reste francophone.» (pp.273-274)

On observe le rapprochement d'Irma avec Karl. Il existe des mots de leurs langues ainsi que Alsace et Algérie. Les pays mentionnés ont connu le même sort, on explicite le sens de mot Alsagérie :

"Alsace, Algérie ; les deux mots tanguaient soudain. Elle leur trouva une résonance commune, une musique qui semblait les accoupler, à moins que ce ne fût plutôt une même blessure ancienne, des cicatrices en creux qui, conjuguées, risqueraient de réapparaître... Oui, vraiment, une algie sourde les reliait : Alsace, Algérie. Irma murmura, du bout des lèvres, ces deux noms de pays, de terroir noir, lourd d'invasions, de ruptures ou de retours amers... » (p.285) 
La langue a pour but de réunir, assembler et unifier. L'allemand est une langue qu'Irma a apprise, ce n'est donc pas sa langue maternelle. Il est possible de préciser que la langue sert à relier Irma à ses parents qu'elle n'avait jamais connus :

« J'ai appris l'anglais, après mon français maternel et l'allemand - c'était cette dernière langue, pensais-je, qui me reliait à ces parents assassinés que je n'avais jamais connus! » (p.302)

\section{La question de la langue chez Thelja et François}

Thelja appelle « l'étranger» parce qu'on lui trouve une qualité, une impersonnalité. En outre, elle n'ose pas l'appeler par son nom pour ne pas déchiffrer tout. L'impersonnalité occupe une place centrale et qui a de l'importance pour elle parce qu'il s'agit de ne pas appartenir à n'importe qui et n'importe où. Au lieu d'être subjectif, on choisit d'être objectif et on est à la recherche d'un étranger absolu :

" “D’un étranger ?” me répondrez-vous toutefois si du moins ce soir, je me livre. Je serai contrainte, par besoin de vérité, de préciser quelque peu gênée - Enfin, le regard... d'un Français ! Puis je me rassurerai, me disant à moi- même, à demi interrogative, c'est un regard d'homme, est-ce un regard pur ? » (p.45)

On préfère qu'il soit un étranger pour ne pas partager des mots, pour ne pas parler avec des langues différentes et avec des langues qui leur rappellent la notion de l'ennemi. Il est possible de préciser qu'il y existe la langue incommunicable. Le silence règne entre eux quand on prend en considération l'étranger en négligeant sa nationalité à travers la langue :

" "Peut-être qu’il m'ennuierait- se remit-elle à penser ardemment, comme on court, comme on fuit, pour précéder le temps, pour... - à Paris, non, mais ici, je préférerais par moments qu' 'il soit totalement étranger ; on ne pourrait échanger des mots; seulement des caresses /... '’Soudain, elle comprit qu'elle avait envié, ce matin même, l'état de Hawa amoureuse. » (p.80)

Thelja parle de son amant à Eve. Elle l'appelle « un étranger » et explique la raison pour laquelle elle le nomme ainsi. Un étranger est quelqu'un qui n'a pas la même langue maternelle qu'Eve. C'est pour cette raison qu'elle ne peut pas se donner entièrement à un étranger. L'étranger se voit comme un seul espoir pour s'enfuir du passé douloureux : 
" “Un étranger? C'est-à-dire quelqu'un que je ne pourrai aimer ainsi, au creux de cette beauté de ma langue d'enfance !... Me retrouver au plus profond de moimême, en me donnant, en m'anéantissant !... Oui, un étranger, pourquoi ai-je d'abord défini ainsi l'amant de ces nuits ?”. » (p.107)

Thelja étudie sur le manuscrit qui est détruit pendant la guerre, sauvé du feu et utile pour sa thèse. Pendant sa recherche, elle s'adresse à des différentes langues ainsi que le latin, le français et l'allemand. Elle fait plus attention car le texte est en latin, pour le comprendre mieux, il faut se débrouiller. Bien que l'allemand ralentisse son travail de temps en temps, elle inscrit la traduction française pour accéder à l'ultime compréhension qui nécessite une compréhension profonde par le biais de la langue. Thelja ressemble à ce texte parce qu'elle est nomade comme ce manuscrit :

«Une heure après, elle se trouve plongée dans la contemplation d'un chef- d'œuvre ; du moins de sa copie, car l'original est perdu, hélas ! Elle recopie des vers en latin ; en face, elle y inscrit la traduction française. Quelquefois, des expressions et des notes en allemand, qui parsèment le texte, ralentissent son travail. » (p.99)

On peut bien préciser que le silence est lié à la question du langage. Leur amour les rend muet, silencieux et tranquille. La langue perd sa fonction et donne place au silence qui règne entre les amoureux. Des fois la communication ne suffit pas, il faut les gestes, les implicites et le silence pour comprendre quelqu'un qui se trouve en face de nous ou bien pour vérifier si le message est transmis :

«Comment, ces trois premiers jours, à Rotterdam, nous nous sommes aimés ? Je ne me souviens pas, ou plutôt j'en suis sûre : sans les mots, en dehors des mots, lui et moi soudain muets... La stupéfaction, le trouble amoureux rend muet. » (p.69)

Thelja choisit le silence. Au moment où son silence finit, elle emploi des mots précis afin de s'entendre. Les mots sortent de la langue et s'envolent au-delà des gens pour qu'on soit en harmonie avec l'autre :

«Un silence dans la chambre. François fume. Thelja épie les traits de celui qui a parlé, “alors que sans doute, se dit- elle, il a parlé surtout pour s'entendre, une 
fois au moins, après cinquante ans de mutisme. Il a parlé pour mettre des mots précis sur tant d'images, tant de fantômes aussi !” »(p.129)

Thelja est à la quête d'un étranger parce qu'elle voudrait un homme qui est sourd au sens figuré. Elle voudrait qu'il ne l'entende pas quand elle avoue son passé. Afin de maintenir leur relation, l'étranger doit devenir nomade et prêt à partir à chaque fois comme elle :

« Je suis venue jusqu'ici pour toi, pas seulement pour toi !... C'est aussi parce que je passe mes nuits avec un homme, un étranger. » (p.105)

Thelja se demande et pose des questions par rapport à la langue. L'obscurité règne, ils peuvent s'aimer en muets. Pour Thelja, l'homme idéal, c'est quelqu'un qui ne parle pas beaucoup. C'est pour cette raison qu'elle pourrait l'aimer même s'il ne parle aucune des langues qu'elle parle :

«- Si je ne parlais ni arabe ni français, murmura-t-elle tandis qu'il l'installait au creux de son épaule. Elle reprit : Si je ne parlais ni arabe ni français, si, toi, tu ne parlais ni français ni... disons allemand' ni alsacien, est-ce que nous nous aimerions toutes ces nuits de la même façon?...

L'une ne parlerait aucune des langues que je comprends. Et je t'aimerais d'emblée, tout autant ! Je te ferais réciter des vers de ta langue qui me serait indéchiffrable, un babil, un parler d'oiseau...» (pp.224-225)

François a traversé Strasbourg avec sa mère pour retrouver le père. L'entourage est crucial en apprenant une langue parce qu'on pourrait la chance ou bien le malheur d'apprendre plusieurs langues. On le considère comme être en paradis ou en enfer :

«Je me souviens ensuite des jours d'automne à Oberhof-fen. Grand-Père s'occupait de moi, chaque matin : il m'apprenait à lire, en allemand et en français... Il avait été enfant et adolescent peu avant 1900, sous la première occupation allemande... A la maison, il parlait alsacien. »... son fils ne comprenant rien, accroché seulement à elle dans cette marche qui devient interminable... (p.130)

En raison du bonheur, elle parle la langue maternelle au lieu de parler français. Il existe la mémoire de la douleur :

«Ces derniers mots, Thelja ne les comprit pas ; elle supposa que c'était un chant alsacien qui terminait cette évocation matinale... » "Elle chantonne, et pas en français ; dans sa langue maternelle probablement. » (p.133) 
Il s'agit de la langue de la bouche et de la langue de communication. Les deux sont liés l'un à l'autre car pour pratiquer la langue, il faut utiliser la langue de la bouche. On y observe l'importance des langues à travers les mots utilisés. En fait, c'est l'exemple de l'importance dramatique de la question de la langue :

«- Neige ! a appelé François, à mi-voix.

- Neige?

- $\quad$ Moi qui te connais depuis vingt- cinq ans au moins, jamais - et je croyais comprendre alors assez d'arabe! -, jamais, je n'ai pensé que ton prénom (elle prend affectueusement son amie par l'épaule) avait un sens... Et, celui-là !

La discussion dériva sur l'existence de la neige en Algérie : en Kabylie, dans les Aurès... Eve reprend : Thelja, c'est en fait un prénom bien rare ! J'ai toujours cru qu'il était berbère, pas arabe! » (p.173)

Malgré les différentes cultures, François ne sépare pas de son amour. Il voudrait apprendre la signification de son prénom Thelja :

«Comment te le répéter sans savoir la signification première? ».

Thelja et François n'ont pas la langue commune. Cette situation les fascine et les éloigne. Même si les amoureux ne connaissent pas leur langue, leur pays et leurs coutumes, ils s'attirent l'un l'autre, ils peuvent se plaire parce que l'amour ne vise ni la logique ni le but :

«Tu ne parlerais aucune des langues que je comprends. Et je t'aimerais d'emblée, tout autant! Je te ferais réciter des vers de ta langue qui me serait indéchiffrable, un babil, un parler d'oiseau... Un bruit, non, une musique. » (224-225)

Selon Thelja, le français est une langue qui porte le sang de son père qui est mort pendant la guerre. Elle se ferme la langue car elle ne voudrait pas se souvenir des conflits provenant de langue : 
" “Où se tapit la langue, dans tout cela ?” s’interrogea-t-elle..., l'homme français, mais dans un parler ensauvagé de l'autre bout de la terre!

"Où se tapit la langue, dans tout cela ? se redit-elle, entêtée. Eh bien, elle se ferme, la langue! (pp.227-228)

L'histoire de guerre entre les deux pays complique la relation amoureuse de Thelja et François. Thelja n'est pas encore certaine en quelle langue elle va parler et elle choisit de tutoyer. Elle n'est pas consciente de ce qu'elle dit pendant sa relation avec François. Cette œuvre nous montre que quelques dialectes ont des limites occidentales :

«Qu'ai-je dit de moi à toi, mon amour, enfin je te tutoie nuit et jour et je ne sais plus en quelle langue je te parle, ni celle de ma terre - la langue nouée et rauque dans laquelle, une fois pour toutes, ma mère s'est emmaillotée-, pas davantage celle que je partage avec Halim, celle avec laquelle il me caressait et grâce à laquelle je l'avais aimé, car ce dialecte d'Oran et des limites occidentales, c'était comme si nous jubilions ensemble, » (p.345)

La langue inventée perd son origine. Thelja préfère vouvoyer car François est plus âgé qu'elle. En plus, Thelja parle en français bien qu'elle ne soit pas sûre de quelle langue elle va parler. Il n'y a pas d'homme sans culture sans nationalité, chaque homme nait dans une culture appartenant à une nationalité et en fait partie. On se nourrit de cette culture existante :

«Dans quelle langue, François, je vous parle, si c'est en français comme à Paris, c'est normal, je vous vouvoie : vous avez vingt ans au moins de plus que moi, je pourrais être votre fille mon père vivant aurait votre âge, ou un peu plus. » (p.346)

Le mot Alsagérie vient de naitre au cours de la dernière nuit entre Thelja et François. La prononciation de mot Alsagérie pose un dilemme : le mot concerné vient de la langue de Thelja ou bien celle de François. La citation ci-dessous nous montre que le rêve est lié à la langue :

- $\quad$ Alsace, Algérie... Non,plutôt Alsa-gérie /

- $\quad$ Alsagérie, en quelle langue ce mot ? Dans la tienne, dans la mienne ?

- Al za gé rie /

- Ce mot, il tangue !

Dis-le maintenant à ton tour. Je me souviens, il y a longtemps, ou un jour à venir, peut-être- dans un lointain, dans un venir de l'avenir, je me souviendrai, en tout 
cas- dans l'un de mes rêves dont il ne me reste souvent qu'un bruit, à l'aube - toi, oui toi, tu apprenais ma langue !... Alors tu aurais dit, si nous l'avions inventé- ni chez toi, ni chez moi, ou dans les deux parlers à la fois : "el za djê rie” ! (p.372)

Les amoureux découvrent différentes nuances quand chacun épèle le mot dans sa langue maternelle car chaque langue a son articulation propre. C'est pour cette raison que chaque mot dans chaque langue a un signifiant et un signifié :

Le z dans mon alphabet d'enfance n'est pas pourtant une trace de souffrance, non. Cette consonne annonce la beauté et l'éclat : z comme “zina”. Zina, l'adjectif signifie belle; comme substantif, il désigne l'accouplement. Il y a donc un couple dans "Alsagérie", un couple heureux; un couple faisant l'amour. Comme nous, à présent, dans cette pénombre, devant la fenêtre ouverte... (p.373)

Ils ont inventé un mot qui résume leur histoire, leur relation pour que ce mot ne les ruine pas en rappelant l'histoire traumatisante :

\section{- $\quad$ "Alsagérie" : palpe mes lèvres quand je redirai ce mot qui nous}

résume... Tes doigts me connaissent, me regardent ! (p.374)

Le choix de tutoyer et vouvoyer se différencie de la personne en personne. Il préfère tutoyer alors qu'elle préfère vouvoyer. C'est peut-être en raison de la distance entre eux. Ils s'adressent à leur guise et en prenant compte le respect envers l'autre qui reste toujours le seul espoir :

«La seconde fois, il vint exprès pour elle : il lui demanda, quelques jours auparavant, au téléphone, se remettant à la tutoyer comme du temps de leurs jours à Strasbourg: “je t'en prie, essaie de te libérer, cette fois, pour... pour nous !” Elle hésita, se tut une seconde, puis répondit très vite : "je verrai ! Je vous laisserai un mot à votre hôtel !’ Elle raccrocha : peu de temps auparavant, elle lui avait annoncé qu'elle prenait un travail de secrétariat, à mi-temps, pour l'été. » (p.388)

Le choix de langue est un thème essentiel qui se situe au centre de l'œuvre. Elle finit par signer en arabe. On essaie d'établir un pont entre soi et l'autre à travers la langue :

« Je passe chaque jour par là ; je vous indique cela de mémoire. Au bout du quai, côté Seine, se trouve un banc de pierre. Attendez-moi là, le premier jour de votre arrivée de préférence, à dix-sept heures (je risquerai de vous faire attendre cinq 
minutes, ou dix)... et, tant pis, si, par malheur, il pleut." Elle signa en lettres arabes. " (p.389)

L'intérêt que François porte au nom de Thelja est si sincère qu'il le pousse à le dire dans sa propre langue pour traduire la flamme qu'il ressent pour elle. Il s'adresse avec tout son cœur car la vie est significative avec elle. Leur relation résume un univers à admirer. Même s'ils ne connaissent pas leur langue, ils se regardent et peuvent s'amouracher l'un de l'autre :

«O neige, soupire-t-il, femme ardente qui me brûle! ».

Neige n'est pas un hasard. La neige est liée aux souvenirs de Thelja car sa mère a dû marcher dans la neige pour rejoindre son mari. Puisqu'elle est enceinte à ce temps-là, on attribue le prénom « neige » à Thelja :

«De fait, François, assis sur ce banc de pierre, sans lâcher les mains de Thelja, François semblait presque expansif. Avec comme une espérance inattendue.

- $\quad$ Neige... Oh oui, ma Neige brûlante, tu me manques !

Elle avait doucement libéré ses mains. Il chercha à l'embrasser. Attira, de son bras qu'il venait de glisser sous la chevelure de Thelja (elle avait laissé pousser ses cheveux), le visage de la jeune femme. Elle résista à peine. » (p.392)

Thelja signe sa dernière lettre en arabe. Cela peut prouver que c'est un indice pour retourner à la langue maternelle ou bien c'est un indice qu'elle est incapable de s'exprimer aisément dans la langue de l'ennemi :

« en escargot de la lanterne, je braverai le premier vent d'avant l'aurore, immobilisée en plein ciel, au sommet de la flèche de lumière, immense doigt dressé sur le plus haut toit de l'Europe.

Je ne redescendrai pas : après la nuit et juste avant le jour, le vide règne là-bas, debout, un cri dans le bleu immergé... » (p.405)

On peut bien conclure que Thelja choisit le silence et l'étrangeté absolue contrairement à Eve qui cherche un compromis linguistique. Chaque personnage fait de son mieux et coupe les cheveux en quatre pour s'en sortir. Ils se débrouillent, la langue affecte différemment leur relation. Il est possible de mentionner que la langue et la culture sont liées et se basent sur la problématique de l'impérialisme à part le multilinguisme et multiculturalisme. Il vaut mieux de préciser que la langue est une pointe de carrefour des tous les langues d'où vient l'internationalisme. Est-ce que la langue qui influence la culture ou bien la culture qui influence 
la langue ? C'est un vrai dilemme. Paradoxalement, il vaut mieux d'exprimer que la langue et la culture se nourrissent l'un l'autre. Il est impossible de les réfléchir séparément.

\section{Conclusion}

Dans cet essai, on a essayé d'aborder la question du langage en se penchant sur l'interculturalité, le multiculturalisme, le silence, le rêve et la recherche d'un étranger. Les couples essaient de trouver la paix via les langues. Il s'agit des couples mixtes. Les couples appartiennent aux différentes nations. Cette situation ouvre un nouvel horizon pour l'échange et pour la reconnaissance. L'interculturalité est une autre notion qui est en question. En plus, on traite de multiculturalisme. Les couples arrivent à trouver un point d'équilibre dans leur vie d'exil.

L'œuvre bâtit un pont pour passer d'une langue à une autre et aussi d'une nation à une autre. Il est possible de préciser que les frontières peuvent être franchies. On prend en considération les langues qui effectuent l'échange culturel. Pour unir des langues, on crée un espace nouveau «Alsagérie ». Grâce aux langues mélangées, les véritables échanges trouvent leurs commencements. L'ouverture sur autre langue permet d'ouvrir une nouvelle fenêtre et de ne pas se quereller avec les gens appartenant à des cultures différentes.

Les couples et leur relation varient d'une personne à l'autre. Par exemple, Eve et Hans reflètent une partie optimiste et plus positive que l'autre couple. Alors que Thelja et François reflètent une partie plus pessimiste, instable, troublée, aphasique et nomadique. On a essayé de mentionner comment leur rapport à la langue affecte différemment leur relation à l'homme, leur relation à Strasbourg tout au long de ce travail.

A la lumière de tout ce qu'on vient de citer jusqu'ici, on devrait répondre à la question suivante : est-ce que les couples voudraient rester tel qu'ils étaient ou bien devenir d'autres individus ? Le problème est de savoir si ce sont les couples qui choisissent la langue ou bien si c'est la langue qui choisit les couples. Est-ce que la langue qui influence la culture ou bien la culture qui influence la langue ? C'est un vrai dilemme. Il est difficile de donner la réponse à ces questions. Chaque lecteur devrait répondre à son opinion après avoir lu cet œuvre. Les langues sont indispensables pour émettre les idées. De plus, elle a pour but de réunir. De même, les langues sont considérées comme des symboles d'identité et comme marqueur d'identité. C'est pour cette raison que la langue entretient des rapports avec la culture et l'identité.

On y retrouve un triangle linguistique : français, allemand, alsacien. On essaie d'établir un pont entre soi et l'autre à travers la langue quoi que l'autre langue soit considérée comme une langue de l'ennemi. La langue est perçue comme un obstacle, il faut franchir les pièges, il 
faut les écarter afin qu'on survive. Sans la langue, on perd la personnalité. Il est impossible de vivre en absence d'une langue. C'est pour cette raison que la langue a une fonction d'unir les individus sous l'aspect de les allier consciemment. Sans la langue, la vie serait donc une erreur.

On peut conclure que la langue est utilisée pour fortifier la liaison, pour enrichir l'entourage et pour améliorer l'identité personnelle. Elle sert à fumer le calumet de la paix tout en enterrant la hache de guerre afin de se réconcilier.

\section{Bibliographie}

- Djebar, A. (1997). Les Nuits de Strasbourg. Actes Sud.

- Habchi, F.Z. (2009). Au-delà des frontières littéraires : Les Nuits de Strasbourg de Assia Djebar, Synergies Algérie n 5 - 2009 pp. 201-208.

- MACK, C. (2005/2006). A la rencontre de l'Autre : l'écriture de l'altérité dans Les Nuits de Strasbourg d'Assia Djebar, Wissenschaftliche Arbeit für Lehramt Gymnasium /Mémoire de Master 2.

- Nakkouch, T. (2012). Les nuits de Strasbourg: Assia Djebar écrit la migration maghrébine et crée de nouvelles sphères pour la langue française, Université Ibn Zohr, Maroc, Francosphères, vol. 1, no. 2. 